\title{
A Survey on to Enhance Security Approach Using Discretized Centralization for Captcha as Graphical Password
}

\author{
Bhavana A. Barbole ${ }^{1}$, Shubhangi Surywanshi ${ }^{2}$ \\ ${ }^{1}$ Master of Computer Networking, Savitribai Phule Pune University, G. H. Raisoni Collage of Engg and Technology, Wagholi, Pune, India \\ ${ }^{2}$ Assistant Prof, Department Of Computer Engineering, Savitribai Phule Pune University, G. H. Raisoni Collage of Engg and Technology, \\ Wagholi, Pune, India
}

\begin{abstract}
CAPTCHA is an acronym for Completely Automated Public Turing Test to distinguish Computers and Humans One from the other. Captcha is one of the broadly utilized systems for keeping malignant project from getting to the web asset naturally. Presently a day's for web security there exists distinctive kind of Captcha, for example, Content Captcha, Picture Captcha, Sound Captcha and Video Captcha. In this paper online security plan is built with content and graphical passwords. Captcha and Graphical passwords are coordinated and a novel group of graphical secret word frameworks based on top of Captcha innovation is called as Captcha as graphical passwords (CaRP). The CaRP plan is upgraded with more assault taking care of components that enhances the level of security in online application framework furthermore gives better verification.
\end{abstract}

Keywords: Captcha, Graphical password, Authentication, Security, CaRP

\section{Introduction}

Today, confirmation is the chief technique to ensure data security and the most widely recognized and advantageous strategy is secret key verification. Conventional alphanumeric passwords are series of letters what's more, digits, which are simple and well known to basically all clients. In any case, there are a few inborn deformities and insufficiencies in alphanumeric passwords, which effectively develop into security issues. Because of the restriction of human memory, most clients have a tendency to pick short or straightforward passwords which are anything but difficult to recall. In addition, alphanumeric passwords are helpless against shoulder surfing attack, spyware attack and social designing attack and so forth. Persuaded by the guarantee of enhanced watchword ease of use and security, the idea of graphical passwords was proposed in 1996.

The primary objective of graphical passwords is to use pictures or shapes to supplant content, subsequent to various Subjective and mental studies exhibited that individuals perform far superior when recollecting pictures than words.

There are 4 existing graphical secret word plans:

a) Drawmetric schemes

b) Locimetric schemes

c) Cognometric schemes

d) Hybrid schemes [1]

Countless watchword plans have been proposed. They can be grouped into three classifications as indicated by the errand included in learning and entering passwords: acknowledgment, recognition, and demonstrated review. A recognition-based scheme requires identifying among decoys the visual objects belonging to a password portfolio. A remembrance-based scheme requires a user to regenerate the same result without cueing. Draw-A-Secret (DAS) was the first recall-based scheme proposed. In an Indicted review conspire; an outer signal is given to help and bots in Unravelling certain hard AI problems.[2]

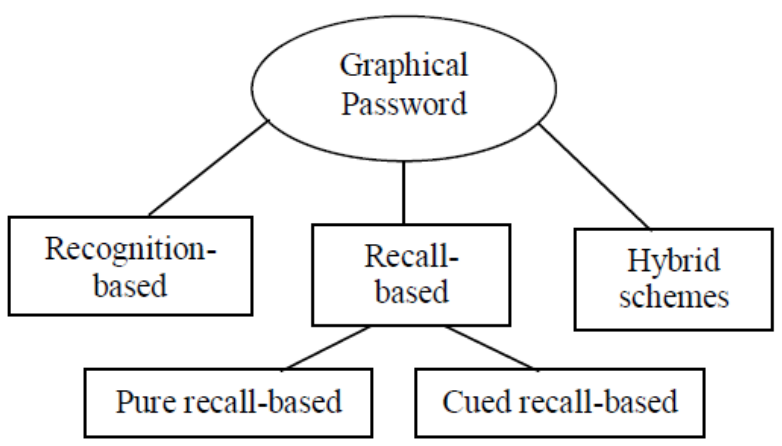

Figure 1: Categorization of Graphical password authentication techniques [3]

Security is critical variable in today's world. It is fundamental for getting to secret information and security parameters were done in view of the cryptography and numerical estimation. In this paper its state around two level of confirmation system which is unique in relation to existing procedures. Cryptography depends on the numerous encryption and decoding calculations. AI (counterfeit consciousness) used to make a hard security challenges. It utilizes the captcha methods to give the security on client interface.

\section{CAPTCHA}

The Captcha relies on upon the gap of abilities in the middle of people and bots in settling certain hard AI issues. It contains two sorts of visual Captcha (i.e.) content Captcha and Image-acknowledgment Captcha (IRC). The past depends on character acknowledgment while the last relies

\section{Volume 4 Issue 11, November 2015}




\section{International Journal of Science and Research (IJSR) \\ ISSN (Online): 2319-7064}

Index Copernicus Value (2013): 6.14 | Impact Factor (2014): 5.611

on upon acknowledgment of non-character articles. Security of content Captcha's has been generally concentrated.

\subsection{CAPTCHA in Authentication}

This method was introduced in to use both Captcha and password in a user authentication protocol, which we will call as Captcha-based Password Authentication (CbPA) protocol, helps to defy the online dictionary attacks. The CbPA-protocol in order to solving a Captcha challenge after inputting a suitable pair of user ID and password unless a valid browser level cookie was received. For an invalid pair of user ID and password, the user has a certain level of probability to solve a Captcha challenge before being to deny their access. An Improved CbPA-protocol is wished-for to storing cookies only on the user-trusted machines and applying a Captcha challenge only when the number of failed login attempts for the specific account has exceeded a threshold limit.

\subsection{Captcha as a graphical password}

\section{Overview of CaRP}

CaRP (Captcha as gRaphical Passwords). CaRP is clickbased graphical passwords, where a sequence of clicks on an image is used to derive a password. Not at all like other snap based graphical passwords, pictures utilized as a part of $\mathrm{CaRP}$ are Captcha challenges, and another $\mathrm{CaRP}$ picture is produced for each login endeavor. The thought of CaRP is straightforward however non specific. CaRP can have various instantiations. In principle, any Captcha plan depending on various item order can be changed over to a CaRP plan. CaRP offers assurance against online word reference assaults on passwords, which have been for long time a noteworthy security risk for different online administrations. This risk is across the board furthermore, considered as a top digital security hazard [3].

Safeguard against online word reference assaults is a more unpretentious issue than it may show up. CaRP likewise offers insurance against transfer assaults, an expanding risk to sidestep Captchas insurance, wherein Captcha difficulties are handed-off to people to unravel. CaRP is powerful to shoulder-surfing assaults if consolidated with double view advancements. CaRP requires understanding a Captcha challenge in each login. This impact on usability can be mitigated by adapting the CaRP image's difficulty level based on the login history of the account and the machine used to $\log$ in. [8] On the basis of the memory tasks in memorizing and entering a password, classification of CaRP schemes can be done as follows:

Recognition based and recognition-recall. The second scheme i.e. recognition - recall CaRP is a new category which works by recognizing an image and using the recognized objects as cues to enter a password. Recognitionrecall combines the tasks of both recognition and cued recall. It retains the advantages of both schemes i.e. recognition advantage of being easy for human memory and the cuedrecall advantage of a large password space [5].

\section{Recognition based CaRP}

\section{a. ClickText}

ClickText is a recognition-based CaRP scheme built on top of text Captcha. Its alphabet comprises characters without any visually-confusing characters.

\section{b. ClickAnimal}

ClickAnimal is also a recognition-based CaRP scheme. It has an alphabet of similar animals such as dog, horse, pig, etc. The password in this scheme is a sequence of animal names such as $\rho=$ Cat, Dog, Horse, Turkey...". One or more models are built for every animal. The CAPTCHA era process wherein $3 \mathrm{D}$ models are utilized to get $2 \mathrm{D}$ models by applying distinctive perspectives, hues, lightning impacts, compositions, and alternatively mutilations are utilized for creating the ClickAnimal picture. The subsequent 2D creatures are then orchestrated on a messed foundation like meadows. A few creatures may be covered by other creatures in the picture, yet their center parts are not covered with the end goal people should distinguish each of them. The quantity of comparable creatures is a great deal not exactly the quantity of accessible characters. ClickAnimal has a littler letters in order, and in this way a little secret key space, than ClickText.

\section{c. AnimalGrid}

Keeping in mind the end goal to oppose human speculating assaults, a sufficiently large powerful secret key space ought to be available for CaRP plans. On the off chance that the ClickAnimal plan be joined with grid based graphical passwords; its secret word space can be expanded. The network can be made relying upon the span of the chose creature. For confirmation prepare, a ClickAnimal picture is shown first. After a creature is chosen, a picture of $n \times n$ network shows up, with the lattice cell size leveling with the bouncing rectangle of the chose creature. Every network cell is marked to offer clients some assistance with identifying [5].

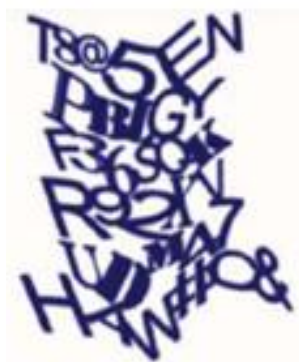

Figure 2: ClickText image with 33 characters

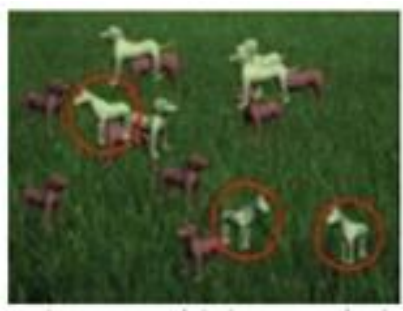

Figure 3: Captcha Zoo with horses circled red 

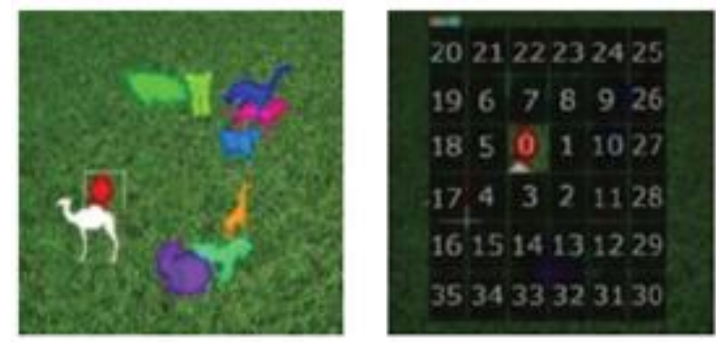

Figure 4: ClickAnimal image (left) and 6 x 6 grid (right) determined by red turkey's bounded rectangle

\section{RECOGNITION-RECALL CaRP}

In recognition-recall CaRP, a password is a sequence of some invariant points of objects. An invariant point of an object (e.g. letter $A$ ") is a point that has a fixed relative position in different incarnations (e.g., fonts) of the object, and thus can be uniquely identified by humans no matter how the object appears in CaRP images. It contains two schemes [8]

\section{A. TextPoints \\ B. TextPoints4CR}

\section{Literature Survey}

\section{A Survey on the Use of Graphical Passwords in Security}

Starting around 1996, various graphical secret key plans have been proposed, persuaded by enhancing secret key ease of use and security, two key elements in secret key plan assessment. In this paper, concentrate on the security parts of existing graphical secret key plans, which not just gives a basic presentation of assault strategies additionally expects to give a top to bottom investigation with particular plans. The paper first sorts existing graphical secret word plans into four sorts as indicated by the verification style and gives a far reaching presentation and investigation for every plan, highlighting security angles. At that point we audit the known assault techniques, order them into two sorts, and compress the security reported in some client investigations of those plans.[1]

\section{Captcha as Graphical Passwords-A New Security Primitive Based on Hard AI Problems}

Numerous security primitives depend on hard scientific issues. Utilizing hard AI issues for security is developing as an energizing new paradigm, but has been under investigated a novel group of graphical secret key frameworks based on top of Captcha technology, which we call Captcha as a graphical passwords (CaRP).. CaRP addresses a, for example, online speculating assaults, transfer assaults, and, if joined with dual-view advancements, shoulder-surfing assaults. CaRP additionally offers a novel way to deal with location the understood picture hotspot issue in prevalent graphical secret word systems, such as PassPoints, that frequently prompts powerless secret word decisions.[2]
3. A Survey on Different Graphical Password Authentication Techniques

These days, client verification is an essential subject in the field of data security. To uphold security of data, passwords were presented. Content based secret word is a famous verification strategy utilized from antiquated times. However content based passwords are inclined to different assaults, for example, word reference assaults, speculating assaults, animal power assaults, social designing assaults and so on. Various graphical secret key plans have been proposed so far as it enhances watchword ease of use and security. In this paper, they lead a far reaching review of the current graphical watchword systems. They ordered these procedures into four: acknowledgment based, immaculate review based, signaled review based and cross breed approaches.[3]

\section{Graphical Password Authentication Using CaRP}

The security activity work is exceptionally indispensable one in all processing components empowered stage, the work of this task states about execution of Graphical Password Authentication utilizing CaRP (Captcha as gRaphical Passwords). This new security primitive depends on hard AI issues. It is based on both writings based Captcha and picture acknowledgment based Captcha. Here the pictures utilized as a part of CaRP are contorted configuration as like Captcha difficulties. It's a sort of validation reaction test. It addresses the different security based assaults. It guarantees the clients with secured login validation. It fit well with the some down to earth applications.[4]

\section{Novel Method for Graphical Passwords using CAPTCHA}

Digital security is an imperative issue to handle. Different client confirmation strategies are utilized for this reason. It serves to maintain a strategic distance from abuse or illicit utilization of profoundly touchy information. Content and graphical passwords are chiefly utilized for confirmation reason. In any case, because of different imperfections, they are not dependable for information security. Content passwords are unreliable for reasons and graphical are more secured in correlation however are powerless against shoulder surfing assaults. Consequently by utilizing graphical secret key framework and CAPTCHA innovation another security primitive is proposed. We call it as CAPTCHA as gRaphical Password (CaRP). CaRP is a mix of both a CAPTCHA and a graphical secret key plan. In this paper they lead a far reaching study of existing CaRP strategies to be specific ClickText, ClickAnimal and AnimalGrid. Talked about the qualities and confinements of each strategy and point out examination heading here. [5]

\section{CAPTCHA as Graphical Password}

The most widely recognized PC validation technique is to utilize alphanumerical usernames and passwords. This technique has been appeared to have critical downsides. For instance, client tends to pick passwords that can be effectively speculated. Then again, if a secret key is difficult to figure, at that point it is regularly difficult to recall. In this paper, they direct a complete review of the current graphical secret key systems and captcha. Utilizing hard AI issues for security is developing as an energizing new worldview, yet has been underexplored. In this paper, displayed another

\section{Volume 4 Issue 11, November 2015}




\section{International Journal of Science and Research (IJSR) \\ ISSN (Online): 2319-7064}

Index Copernicus Value (2013): 6.14 | Impact Factor (2014): 5.611

security primitive taking into account hard AI issues, graphical watchword frameworks fabricated on top of Captcha innovation, which we call Captcha as graphical passwords (CaRP). CaRP is both a Captcha and a graphical secret key plan .[6]

\section{Graphical Password Scheme Using Discretized Centralization}

Ordinary watchword plans are defenseless against shoulder surfing, numerous shoulder surfing safe graphical secret key plans have been proposed. This paper displays an coordinated assessment of the Persuasive Cued Click-Points graphical secret key plan, including ease of use and security assessments, and execution contemplations. We utilize influence to impact client decision in snap based graphical passwords, urging clients to choose more arbitrary, and henceforth more hard to figure, snap focuses [7]

\section{Security Analysis}

As a system of graphical passwords, CaRP does not depend on the security of any particular Captcha plan. On the off chance that one Captcha plan gets broken; another and more powerful Captcha plan may show up and be utilized to build another CaRP plan. CaRP offers protection against online dictionary attacks on passwords, which have been for long time a major security threat for various online services. Defending against online dictionary attacks is a subtler problem than it might appear.

CaRP makes it much harder for terrible folks to perform computerized theory assaults. Notwithstanding when a human is included, the assault is still costly and backed off.

CaRP additionally offers assurance against hand-off assaults, which have been an expanding danger to online applications ensured by Captcha's. In a hand-off assault, Captcha difficulties are handed-off to people to unravel, with their answers returned. Graphical passwords, albeit other snap based graphical passwords, for example, PassPoints are helpless against such assaults.[9]

\section{Conclusion}

The paper directs a complete review of CAPTCHA as Graphical Password plans. CaRP is a blend of both a CAPTCHA and a graphical secret word plan. CaRP plans are named Recognition-Based CaRP and Acknowledgment Recall CaRP. We have talked about Recognition- Based CaRP which incorporate ClickText, ClickAnimal and AnimalGrid strategies in this paper. Current graphical secret word methods are a distinct option for content watchword yet are still not completely secure. As a structure, CaRP does not depend on any particular CAPTCHA plan. Whenever one CAPTCHA plan is broken, another and more secure one may show up and be changed over to a CaRP plan. Because of sensible security and convenience and pragmatic applications, CaRP has great potential for refinements. The convenience of CaRP can be further enhanced by utilizing pictures of diverse levels of trouble taking into account the login history of the client and the machine used to sign in.

\section{References}

[1] Haichang Gao, Wei Jia, Fei Ye and Licheng Ma,"A Survey On The Use of Graphical Passwords in Security" JOURNAL OF SOFTWARE, VOL. 8, NO. 7, JULY 2013

[2] M.Ramapriya,R.Yamini,Mr.M.KrishnaMoorthy,"Captc ha as Graphical Passwords - a New Security Primitive Based on Hard AI Problems", SSRG-IJMCA - volume 2 Issue 2 March to April 2015

[3] Saranya Ramanan,Bindhu J S, "A Survey on Different Graphical Password Authentication Techniques"IJIRCCE Vol. 2, Issue 12, December 2014

[4] Ragavendra .A, Jeysree .J, "GRAPHICAL PASSWORD AUTHENTICATION USING CaRP", IJARCET, Volume 4 Issue 2, February 2015

[5] Jayshree Ghorpade, Shamika Mukane, Devika Patil, Dhanashree Poal, Ritesh Prasad,"Novel Method for Graphical Passwords using CAPTCHA", (IJSCE), Volume-4 Issue-5, November 2014

[6] Magniya Davis, Divya R, Vince Paul, Sankaranarayanan P N, "Captcha As Graphical Password", IJCSIT, Vol. 6 (1) , 2015,

[7] Zarqa Rehmani, Siddhi Keluskar, Karishma Shaikh, Vaishali Baviskar, " Graphical Password Scheme Using Discretized Centralization", IJCSN, Volume 4, Issue 2, April 2015

[8] Bin B. Zhu, Jeff Yan, Guanbo Bao, Maowei Yang, and Ning $\mathrm{Xu}$," Captcha as Graphical Passwords-A New Security Primitive Based on Hard AI Problems". IEEE TRANSACTIONS ON INFORMATION FORENSICS AND SECURITY, VOL. 9, NO. 6, JUNE 2014891

[9] Bin B. Zhu, and Jeff Yan," Towards New Security Primitives Based on Hard AI Problems"2013.

[10] P. C. van Oorschot, A. Salehi-Abari, and J. Thorpe, Purely automated attacks on passpoints-style graphical passwords," IEEE Trans. Inf. Forensics Security, vol. 5, no. 3, pp. 393-405, Sep. 2010.

[11] P. C. van Oorschot and J. Thorpe, Exploiting predictability in click based graphical passwords," $J$. Comput. Security, vol. 19, no. 4, pp. 669-702, 2011.

[12] M. Alsaleh, M. Mannan, and P. C. van Oorschot, Revisiting defenses against large-scale online password guessing attacks," IEEE Trans. Dependable Secure Comput., vol. 9, no. 1, pp. 128-141, Jan./Feb. 2012.

[13] New CAPTCHA interface design for mobile devices," in Proc. 12th Austral. User Inter. Conf., 2011, pp. 3-8.

[14] M. Motoyama, K. Levchenko, C. Kanich, D. McCoy, G. M. Voelker, and S. Savage, Re: CAPTCHAsUnderstanding CAPTCHA-Solving Services in an Economic Context," in Proc. USENIX Security, 2010, pp. $435-452$.

[15] S. Kim, X. Cao, H. Zhang, and D. Tan, Enabling concurrent dual views on common LCD screens," in Proc. ACM Annu. Conf. Human Factors Comput. Syst., 2012, pp. 2175-2184.

[16] J. Bonneau, The science of guessing: Analyzing an anonymized corpus of 70 million passwords," in Proc. IEEE Symp. Security Privacy, Jun. 2012, pp. 20-25. 
[17] Real User Corporation. The science behind Passfaces. White paper, http://www.realuser.com/published/ ScienceBehindPassfaces.pdf, accessed Feb. 2012.

[18] T. S. Ravi Kiran, Y. Rama Krishna, Combining CAPTCHA and graphical passwords for user authentication", International Journal of Research in IT \& Management, Volume 2, Issue 4 (April 2012) (ISSN 2231-4334)

[19] X. Liu, H. Gao, L. Wang and X. Chang, -ArEnhanced Drawing Reproduction Graphical Password Strategy".Journal of Computer Science and Technology, 26(6): 988- 999. 2011.

[20] R. Biddle, S. Chiasson, and P. C. van Oorschot, Graphical passwords: Learning from the first twelve years," ACM Comput. Surveys, vol. 44, no. 4, 2012. 